Article

\title{
Modeling and Analysis of a Compliance Model and Rotational Precision for a Class of Remote Center Compliance Mechanisms
}

\author{
Lei-Jie Lai * and Zi-Na Zhu \\ College of Mechanical Engineering, Shanghai University of Engineering Science, Shanghai 201620, China; \\ zhuzina@126.com \\ * Correspondence: lailj@alumni.sjtu.edu.cn; Tel.: +86-21-678-74553 \\ Academic Editor: Kuang-Chao Fan \\ Received: 10 October 2016; Accepted: 21 November 2016; Published: 28 November 2016
}

\begin{abstract}
The remote center compliance (RCC) mechanism is of great use for practical designs, especially if a pure rotation about a virtual point is required. The analysis of compliance properties and rotational precision for RCC mechanisms are very important for mechanical design in applications where precision is required. This paper formulates an analytical method for the compliance and rotational precision calculations of a class of RCC mechanisms, combined in parallel with two round beam-based isosceles-trapezoidal flexural pivots. The analytical model of the mechanism is established based on the stiffness matrix method to directly obtain the compliance factors that completely define the elastic response of the mechanism. The rotational precision of the mechanism-That is, the position of rotation center-Is then derived using screw theory and a compliance matrix. The validity of this model is demonstrated using finite element analysis simulation and experimental tests. The results of both simulation and experiment verify that the analytical model has high accuracy and promising practical applications. Moreover, the influences of the geometry parameters on the compliance factors and the center shifts are also graphically evaluated and discussed using the analytical model. The results in this paper provide an effective configuration and analytical method for the design and optimization of RCC mechanisms, and are of great practical significance.
\end{abstract}

Keywords: remote center compliance; stiffness matrix; screw theory; rotational precision; finite element analysis

\section{Introduction}

Recently, high-precision parallel alignment between two surfaces has become an important mechanical problem for pattern fidelity in nanoimprint lithography [1-3] and some electrochemical micro/nanofabrication techniques [4,5]. The parallel misalignment between the template and the substrate can lead to non-uniform microstructures fabricated over a large area [1]. The parallel alignment can be achieved by rotations about the axes that lie in the template surface. If the rotation axes do not lie in the template surface, a coupled lateral shift of the imprint position can be generated during the parallel alignment process [2]. Therefore, the parallel alignment can be considered as tip/tilt motions around two perpendicular axes located in the template surface.

To achieve the alignment motions of the template, the parallel alignment stage with a remote center compliance (RCC) characteristic is increasingly being adopted for its advantages, such as no friction, no wear, no need for lubrication, and compact structure. The RCC mechanism was originally designed as a passive wrist accommodator for automatic assembly to compensate for positioning errors between a machine and a part. At Draper Laboratory, Watson et al. developed a series of 
representative RCC systems for automatic assembly devices [6,7]. Later, several other new types of RCC devices were also developed and used in various applications [8-10]. Some RCC mechanisms are still commercially available from a number of companies.

According to the principle of the RCC mechanism, several orientation stages that borrow features from the traditional RCC mechanisms have been developed for nanoimprint machines. Choi et al. [2] first developed a flexure template stage for nanoimprinting by serially assembling two sets of four-bar-linkage compliant mechanisms. Hiroshima et al. [11] developed an active orientation head with the function of distributed force feedback control. Qu et al. [12,13] developed two types of parallelogram-based compliant remote-center-of-motion stages for active parallel alignment. In terms of the analytical model of RCC mechanism, Ciblak et al. [14] presented an accurate model for the RCC mechanism using screw theory to characterize the interesting properties of remote compliance. Pei et al. $[15,16]$ gave a detailed analysis of rotational precision for leaf-type isosceles-trapezoidal flexural (LITF) pivot used in the one-dimensional RCC mechanism. Su et al. [17] derived a symbolic formulation of the compliance and stiffness matrices for several RCC mechanisms.

In the design of RCC mechanisms, the compliance properties and rotational precision are two important specifications determining the range of motion and precision of the RCC mechanisms, respectively. The rotational precision criterion is especially important for some precision-required applications of the flexural mechanisms [15]. Therefore, this paper focuses on the analysis of compliance properties and rotational precision for a class of RCC mechanisms. This kind of RCC mechanism is combined in parallel with two round beams-based LITF pivots, and can achieve two rotations around two perpendicular axes in the remote plane. An analytical model of the RCC mechanism based on the stiffness matrix method is first established to obtain the close-form equations for compliance factors in all directions. The rotation center locations are then derived using the screw theory to analyze the rotational precision of the RCC mechanism. The results of the analytical model, finite elements analysis (FEA), and experimental tests are all compared to validate the high accuracy of the analytical model, which can be utilized in the design and optimization of the RCC mechanisms.

The main contribution of this manuscript is to establish an analytical model for the calculation of the structural compliances and position of rotation center for a class of RCC mechanisms. The modeling method and corresponding theoretical formulas of the compliances and rotational precision proposed in this paper can accurately reveal the movement and deformation mechanism for the RCC mechanism, providing a useful and accurate reference for the optimal design and manufacture of satisfactory RCC mechanism structures.

\section{Configuration of the Remote Center Compliance (RCC) Mechanism}

The LITF pivot (as shown in Figure 1a) is usually adopted in the one-dimensional RCC mechanism [15]. The end-effector of the LITF pivot can rotate around the the intersection point $O$ of the two beam flexures, which can be considered as the remote center point (as shown in Figure 1b). To enable 3-degrees of freedom (DOFs) rotation, two sets of LITF pivots are combined in parallel to obtain an RCC mechanism with four round beam flexures uniformly spaced around two circles on the end-effector and base at intervals of $\pi / 2$, as shown in Figure 2a. Based on the type synthesis approach — freedom and constraint topology (FACT)—of the parallel flexure system in [18,19], there are four constraint lines (blue) that intersect at a common point, as shown in Figure 3. The combination of the four constraint lines permits three independent rotations. The three independent rotation lines (red) in Figure 3 are the mechanism's freedom topology. Therefore, the RCC mechanism in Figure 2a is a 3-DOFs rotational mechanism, and all three rotations are described with respect to the theoretical remote rotation center $O_{R C}$. In other words, the end-effector can be rotated around the point $O_{R C}$ approximately, when the external loads are applied to the end-effector.

By changing the interval angle of the beam flexures to $2 \pi / 3$, another RCC mechanism configuration can be obtained with triple-beam flexures, as shown in Figure $2 \mathrm{~b}$. A compliant 
mechanism with four-beam flexures is adopted for analysis in this work, and a mechanism with triple-beam flexures can be analyzed using the same method.

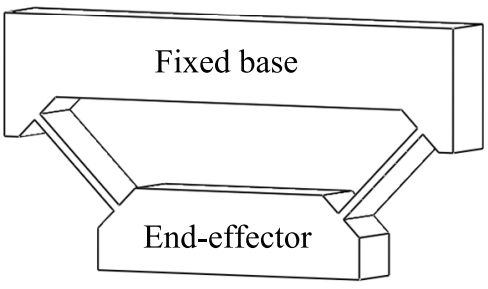

(a)

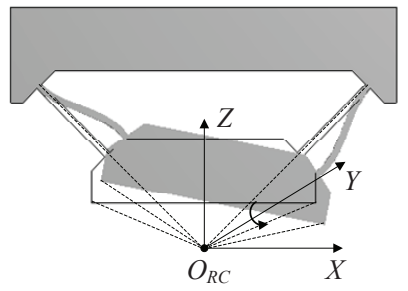

(b)

Figure 1. The leaf-type isosceles-trapezoidal flexural (LITF) pivot. (a) Structure of the LITF pivot; (b) Working principle of the LITF pivot.
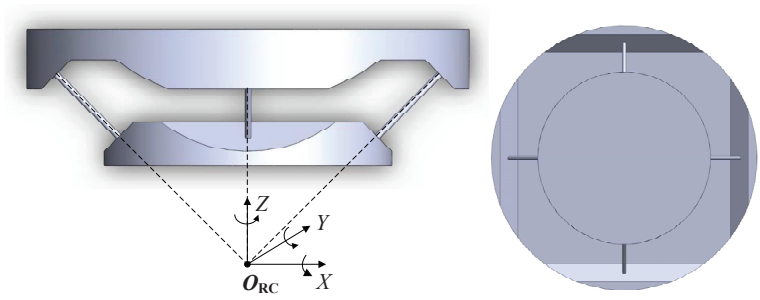

(a)
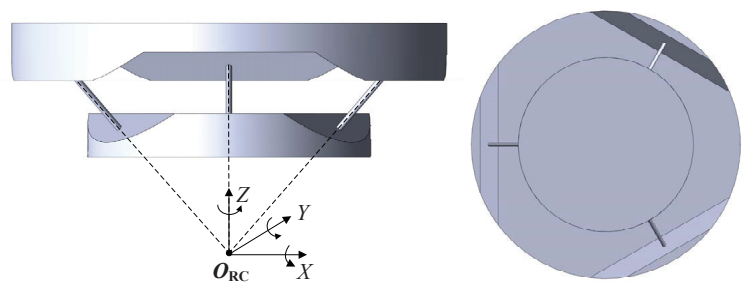

(b)

Figure 2. Two kinds of remote center compliance (RCC) mechanism. (a) The RCC mechanism with four-beam flexures; (b) The RCC mechanism with triple-beam flexures.

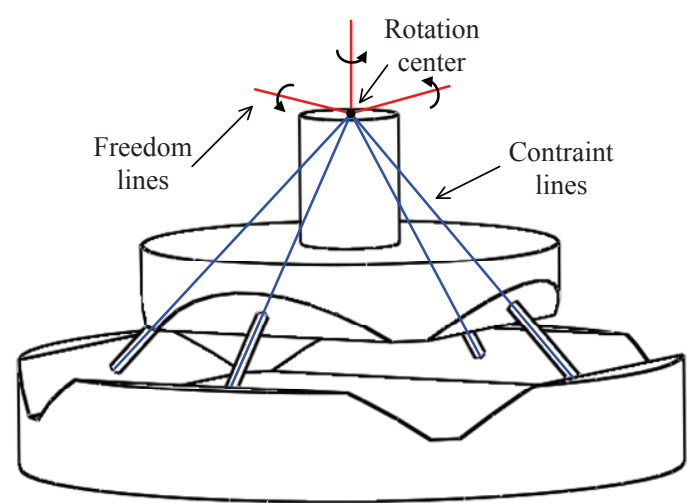

Figure 3. Diagnosis of the RCC mechanism using the Freedom and constraint topology (FACT) method.

\section{Analytical Model}

In this section, we investigate the modeling of the compliance matrix of the RCC mechanism, and also analyze its rotational precision based on screw theory. 


\subsection{Compliance Modeling}

The parallelism alignment between the template and the substrate in the nanoimprint machines is accomplished by the rotational motions around the remote center of the RCC mechanism when a constant contact force between the template and the substrate is applied. Because the parallelism alignment is a quasi-static process, the compliance characteristics that describe the load-displacement relationship of an RCC mechanism are the key input specifications for the design of an RCC mechanism-especially in such static applications. The static analytical model of the RCC mechanism herein is formulated based on the stiffness matrix method [20]. By integrating the compliance matrix of the single beam flexure together with the geometric and material parameters of the RCC mechanism, close-form equations are generated to describe the mechanism's response, and enable further performance analysis and optimization.

The RCC mechanism consists of four individual round beam flexures connected in parallel. In order to obtain the compliance model of the entire RCC mechanism, the compliance matrix of a single beam flexure should be obtained first. The compliance and stiffness matrices for a round beam flexure with the coordinate frame shown in Figure 4 can be described as $[17,21]$

$$
\begin{aligned}
\mathbf{C}^{0} & =\left[\begin{array}{cccccc}
\frac{4 l}{E \pi D^{2}} & 0 & 0 & 0 & 0 & 0 \\
0 & \frac{l^{3}}{3 E I} & 0 & 0 & 0 & \frac{l^{2}}{2 E I} \\
0 & 0 & \frac{l^{3}}{3 E I} & 0 & -\frac{l^{2}}{2 E I} & 0 \\
0 & 0 & 0 & \frac{l}{2 G I} & 0 & 0 \\
0 & 0 & -\frac{l^{2}}{2 E I} & 0 & \frac{l}{E I} & 0 \\
0 & \frac{l^{2}}{2 E I} & 0 & 0 & 0 & \frac{l}{E I}
\end{array}\right] \\
\mathbf{K}^{0} & =\left(\mathbf{C}^{0}\right)^{-1}
\end{aligned}
$$

where $l$ is the length of the beam flexure, and $D$ is the diameter of the round cross-section. $I=\pi D^{4} / 64$ denotes the second moment of the round cross section. $E$ is the elastic modulus, and $G$ denotes the shear modulus of the material. $\mathbf{C}^{0}$ describes the compliance characteristics of free end $O_{i}$ with respect to the other fixed end $O$, as shown in Figure 4.

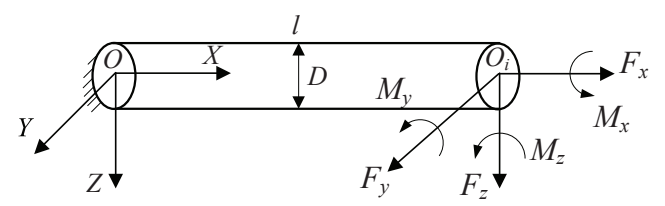

Figure 4. Model of a round beam flexure with specified coordinates.

The coordinate frames of the RCC mechanism are assigned in Figure 5. Because of the small deformation of the flexure mechanism, the original dimensions of the mechanism are used to make a reasonable model of the mechanism. The small deformation assumption is determined by the requirement of the calculation error. For ease of modeling, the origin of the global coordinate system $O-X Y Z$ lies in the centerline of the end-effector, and in the same plane with the ends of the four beam flexures. When an external force is applied to the end-effector of the mechanism, the displacement vector of Point $O$ can be described as $\Delta \mathbf{q}=\left[\begin{array}{cccccc}\Delta x & \Delta y & \Delta z & \Delta \theta_{x} & \Delta \theta_{y} & \Delta \theta_{z}\end{array}\right]^{\mathrm{T}}$. Transforming the displacement vector from $O-X Y Z$ to $O_{1}-X Y Z$ yields

$$
\Delta \mathbf{q}_{1}=\mathbf{J}_{1}^{\mathrm{T}} \Delta \mathbf{q}
$$


where

$$
\begin{aligned}
\mathbf{J}_{1} & =\left[\begin{array}{cc}
\mathbf{I} & \mathbf{0} \\
C\left(\overrightarrow{O O_{1}}\right) & \mathbf{I}
\end{array}\right]\left[\begin{array}{cc}
\mathbf{R}_{y}\left(\angle O / O_{1}\right) & \mathbf{0} \\
\mathbf{0} & \mathbf{R}_{y}\left(\angle O / O_{1}\right)
\end{array}\right] \\
& =\left[\begin{array}{cc}
\mathbf{R}_{y}\left(\angle O / O_{1}\right) & \mathbf{0} \\
C\left(\overrightarrow{O O_{1}}\right) \mathbf{R}_{y}\left(\angle O / O_{1}\right) & \mathbf{R}_{y}\left(\angle O / O_{1}\right)
\end{array}\right]
\end{aligned}
$$

$\mathbf{R}_{y}\left(\angle O / O_{1}\right)$ is the rotation matrix characterized by the rotating angle $-\theta\left(0^{\circ} \leq \theta \leq 90^{\circ}\right)$ of the rotated $O_{1}-X Y Z$ with respect to $O-X Y Z$ around the $Y$ axis, and has the form

$$
\mathbf{R}_{y}(\theta)=\left[\begin{array}{ccc}
\cos (-\theta) & 0 & \sin (-\theta) \\
0 & 1 & 0 \\
-\sin (-\theta) & 0 & \cos (-\theta)
\end{array}\right]=\left[\begin{array}{ccc}
\cos \theta & 0 & -\sin \theta \\
0 & 1 & 0 \\
\sin \theta & 0 & \cos \theta
\end{array}\right]
$$

where $\theta$ is also equal to the geometric parameter of initial inclined angle of the beam flexure, as shown in Figure 5. $\mathrm{C}\left(\overrightarrow{O O_{1}}\right)$ is the skew symmetric matrix corresponding to the position vector $\overrightarrow{O O_{1}}=\left[\begin{array}{lll}r_{x} & r_{y} & r_{z}\end{array}\right]$ expressed in $O-X Y Z$, and can be written as

$$
\mathbf{C}\left(\overrightarrow{O O_{1}}\right)=\left[\begin{array}{ccc}
0 & -r_{z} & r_{y} \\
r_{z} & 0 & -r_{x} \\
-r_{y} & r_{x} & 0
\end{array}\right]
$$

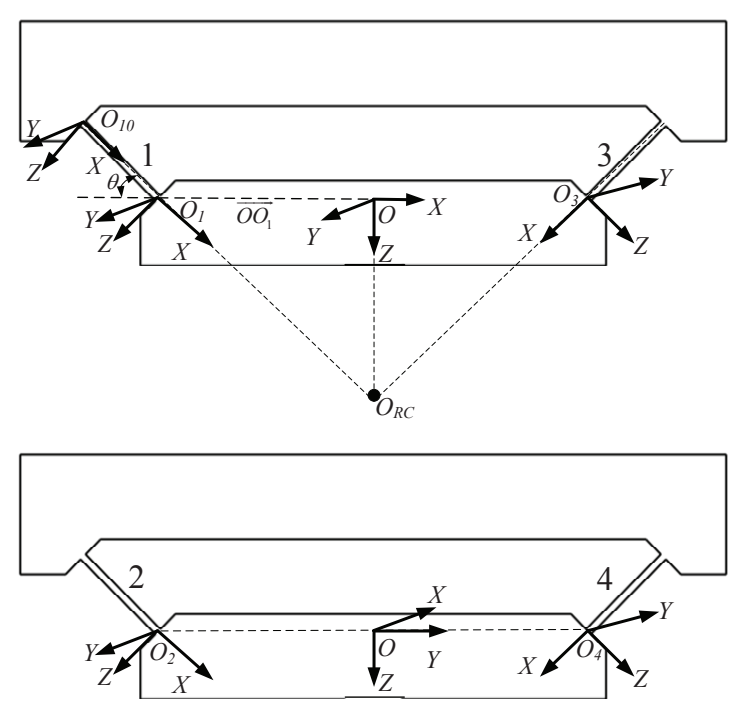

Figure 5. Coordinate system of the RCC mechanism.

According to the displacement vector $\Delta \mathbf{q}$ at Point $O$ and the stiffness matrix of the beam flexure, the $6 \times 1$ force vector $\mathbf{F}_{1}$ applied to $O_{1}$ can be denoted as

$$
\mathbf{F}_{1}=\mathbf{K}^{0} \Delta \mathbf{q}_{1}=\mathbf{K}^{0} \mathbf{J}_{1}^{\mathrm{T}} \Delta \mathbf{q}
$$

Transforming the force vector $\mathbf{F}_{1}$ from $O_{1}-X Y Z$ to $O-X Y Z$ yields the equivalent force vector $\mathbf{F}_{10}$ applied to $O$, which can be represented as

$$
\mathbf{F}_{10}=\mathbf{J}_{1} \mathbf{F}_{1}=\mathbf{J}_{1} \mathbf{K}^{0} \mathbf{J}_{1}^{\mathrm{T}} \Delta \mathbf{q}
$$


There are four beam flexures connecting to the end-effector with a radius of $\left|O O_{1}\right|$ and an interval angle of $\pi / 2$. Hence, the relationship of the force and displacement with respect to the $O-X Y Z$ is derived as [17]

$$
\mathbf{F}=\sum_{i=1}^{4} \mathbf{J}_{i} \mathbf{K}^{0} \mathbf{J}_{i}^{\mathrm{T}} \Delta \mathbf{q}
$$

where

$$
\begin{aligned}
& \mathbf{J}_{2}=\operatorname{diag}\left(\mathbf{R}_{z}\left(90^{\circ}\right), \mathbf{R}_{z}\left(90^{\circ}\right)\right) \mathbf{J}_{1} \\
& \mathbf{J}_{3}=\operatorname{diag}\left(\mathbf{R}_{z}\left(180^{\circ}\right), \mathbf{R}_{z}\left(180^{\circ}\right)\right) \mathbf{J}_{1} \\
& \mathbf{J}_{4}=\operatorname{diag}\left(\mathbf{R}_{z}\left(-90^{\circ}\right), \mathbf{R}_{z}\left(-90^{\circ}\right)\right) \mathbf{J}_{1} .
\end{aligned}
$$

$R_{z}\left(\theta_{z}\right)$ is the the rotation matrix around the $Z$ axis of $O-X Y Z$, which is written as

$$
\mathbf{R}_{z}\left(\theta_{z}\right)=\left[\begin{array}{ccc}
\cos \theta_{z} & -\sin \theta_{z} & 0 \\
\sin \theta_{z} & \cos \theta_{z} & 0 \\
0 & 0 & 1
\end{array}\right]
$$

The symmetric compliance matrix $\mathbf{C}$ of the RCC mechanism is derived as

$$
\begin{aligned}
\mathbf{C} & =\left(\sum_{i=1}^{4} \mathbf{J}_{i} \mathbf{K}^{0} \mathbf{J}_{i}^{\mathrm{T}}\right)^{-1} \\
& =\left[\begin{array}{cccccc}
C_{X}, F_{X} & 0 & 0 & 0 & C_{X}, M_{Y} & 0 \\
0 & C_{Y, F_{Y}} & 0 & C_{Y}, M_{X} & 0 & 0 \\
0 & 0 & C_{Z, F_{Z}} & 0 & 0 & 0 \\
0 & C_{\theta_{X}, F_{Y}} & 0 & C_{\theta_{X}, M_{X}} & 0 & 0 \\
C_{\theta_{Y}, F_{X}} & 0 & 0 & 0 & C_{\theta_{Y}, M_{Y}} & 0 \\
0 & 0 & 0 & 0 & 0 & C_{\theta_{Z}, M_{Z}}
\end{array}\right] .
\end{aligned}
$$

The first subscript in the compliance factors of Equation (11) indicates the deformation about a particular degree of freedom, while the second one points out the load producing the corresponding deformation [22].

\subsection{Rotational Precision Analysis}

According to the principle of the RCC mechanism, the motion of the end-effector is expected to rotate around the fixed center without producing any lateral shifts. However, the actual rotation center does not precisely coincide with the theoretical remote rotation center $O_{R C}$. In order to reveal the rotational precision of the RCC mechanism precisely, the concept of instantaneous rotational center (which could reflect the essential of rigid-body rotation) is adopted as an evaluation criterion of rotational precision [15].

In Equation (11), six column vectors of the compliance matrix are regarded as six screw motions [23]. For instance, when a unit force $F_{X}=1$ is applied to the end-effector at Point $O$, the applied force vector can be written as $\mathbf{F}=\left[\begin{array}{cccccc}1 & 0 & 0 & 0 & 0 & 0\end{array}\right]^{\mathrm{T}}$, and the displacement vector of the $O-X Y Z$ can be calculated as $\left[\begin{array}{llllll}x_{1} & 0 & 0 & 0 & \theta_{1} & 0\end{array}\right]^{\mathrm{T}}=\left[\begin{array}{lllllll}C_{X, F_{X}} & 0 & 0 & 0 & C_{\theta_{Y}, F_{X}} & 0\end{array}\right]^{\mathrm{T}}$, which is the first column vector of the compliance matrix. According to the displacement generated by the applied force $F_{X}$, the homogeneous representation of the rigid body transformation can be given by

$$
\mathbf{g}_{1}=\left[\begin{array}{cc}
\mathbf{R}_{1} & \mathbf{p}_{1} \\
0 & 1
\end{array}\right]=\left[\begin{array}{cccc}
\cos \theta_{1} & 0 & \sin \theta_{1} & x_{1} \\
0 & 1 & 0 & 0 \\
-\sin \theta_{1} & 0 & \cos \theta_{1} & 0 \\
0 & 1 & 0 & 1
\end{array}\right]
$$


This relative motion of a rigid body can be given by the exponential map for a twist $\hat{\xi}_{1}$, which can be represented as [24]

$$
\mathbf{g}_{1}=e^{\hat{\xi}_{1} \theta_{1}}=\left[\begin{array}{cc}
e^{\hat{\omega}_{1} \theta_{1}} & \left(\mathbf{I}-e^{\hat{\omega}_{1} \theta_{1}}\right)\left(\omega_{1} \times v_{1}\right)+\omega_{1} \omega_{1}^{\mathrm{T}} v_{1} \theta_{1} \\
0 & 1
\end{array}\right]
$$

where the twist $\hat{\xi}_{1}$ is

$$
\hat{\xi}_{1}=\left[\begin{array}{cc}
\hat{\omega}_{1} & v_{1} \\
0 & 0
\end{array}\right]
$$

The axis $\omega_{1} \in \mathbf{R}^{3}$ which satisfies $\exp \left(\hat{\omega}_{1} \theta_{1}\right)=\mathbf{R}_{1}$ is

$$
\omega_{1}=\left[\begin{array}{lll}
0 & 1 & 0
\end{array}\right]^{\mathrm{T}}
$$

To find $v_{1}$, we must solve

$$
\left[\left(\mathbf{I}-e^{\hat{\omega}_{1} \theta_{1}}\right) \hat{\omega}_{1}+\omega_{1} \omega_{1}^{\mathrm{T}} \theta_{1}\right] v_{1}=\mathbf{p}_{1}
$$

Expanding the left-hand side of the equation yields

$$
\left[\begin{array}{ccc}
\sin \theta_{1} & 0 & 1-\cos \theta_{1} \\
0 & \theta_{1} & 0 \\
\cos \theta_{1}-1 & 0 & \sin \theta_{1}
\end{array}\right] v_{1}=\left[\begin{array}{c}
x_{1} \\
0 \\
0
\end{array}\right]
$$

The solution is given by

$$
v_{1}=\left[\begin{array}{ccc}
\frac{\sin \theta_{1}}{2\left(1-\cos \theta_{1}\right)} & 0 & -\frac{1}{2} \\
0 & \frac{1}{\theta_{1}} & 0 \\
\frac{1}{2} & 0 & \frac{\sin \theta_{1}}{2\left(1-\cos \theta_{1}\right)}
\end{array}\right]\left[\begin{array}{c}
x_{1} \\
0 \\
0
\end{array}\right]=\left[\begin{array}{c}
\frac{x_{1} \sin \theta_{1}}{2\left(1-\cos \theta_{1}\right)} \\
0 \\
\frac{x_{1}}{2}
\end{array}\right]
$$

Due to the small deformation of the RCC mechanism, the value of $x_{1}$ and $\theta_{1}$ can be regarded as nearly zero. The first element in $v_{1}$ can be calculated as

$$
\frac{x_{1} \sin \theta_{1}}{2\left(1-\cos \theta_{1}\right)}=\frac{x_{1}}{2 \tan \frac{\theta_{1}}{2}} \approx \frac{x_{1}}{\theta_{1}}=\frac{C_{X, F_{X}}}{C_{\theta_{Y}, F_{X}}}
$$

$v_{1}$ can be rewritten as

$$
v_{1} \approx\left[C_{X, F_{X}} / C_{\theta_{Y}, F_{X}} \quad 0 \quad 0\right]^{\mathrm{T}}
$$

Thus, the twist coordinates for $\mathbf{g}_{1}$ are

$$
\xi_{1}=\left[\begin{array}{ll}
v_{1} & \omega_{1}
\end{array}\right]^{\mathrm{T}}=\left[\begin{array}{llllll}
C_{X, F_{X}} / C_{\theta_{Y}, F_{X}} & 0 & 0 & 0 & 1 & 0
\end{array}\right]^{\mathrm{T}}
$$

The pitch $h$ and the rotation axis $l$ of the twist $\hat{\xi}_{1}$ can be calculated as

$$
\begin{aligned}
h_{1} & =\omega_{1}^{\mathrm{T}} v_{1}=0 \\
l_{1} & =\left\{\omega_{1} \times v_{1}+\lambda \omega_{1}: \lambda \in \mathbf{R}\right\} \\
& =\left\{\left[0, \lambda,-C_{X, F_{X}} / C_{\theta_{Y}, F_{X}}\right]^{\mathrm{T}}: \lambda \in \mathbf{R}\right\}
\end{aligned}
$$


The twist coordinates corresponding to the other five column vectors of the compliance matrix can be obtained in the same way:

$$
\begin{aligned}
& \xi_{2}=\left[\begin{array}{ll}
v_{2} & \omega_{2}
\end{array}\right]^{\mathrm{T}}=\left[\begin{array}{llllll}
0 & C_{Y, F_{Y}} / C_{\theta_{X}, F_{Y}} & 0 & 1 & 0 & 0
\end{array}\right]^{\mathrm{T}} \\
& \xi_{3}=\left[\begin{array}{ll}
v_{3} & \omega_{3}
\end{array}\right]^{\mathrm{T}}=\left[\begin{array}{llllll}
0 & 0 & 1 & 0 & 0 & 0
\end{array}\right]^{\mathrm{T}}
\end{aligned}
$$

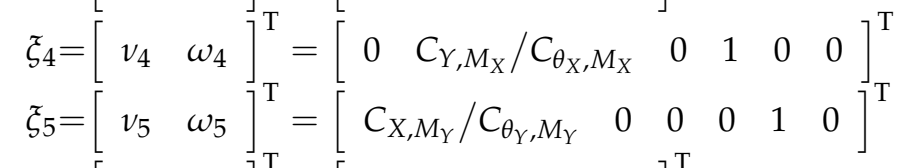

$$
\begin{aligned}
& \xi_{6}=\left[\begin{array}{ll}
v_{6} & \omega_{6}
\end{array}\right]^{\mathrm{T}}=\left[\begin{array}{llllll}
0 & 0 & 0 & 0 & 0 & 1
\end{array}\right]^{\mathrm{T}}
\end{aligned}
$$

All the other pitches can be obtained as

$$
\begin{aligned}
& h_{2}=\omega_{2}{ }^{\mathrm{T}} v_{2}=0 ; h_{3}=\omega_{3}{ }^{\mathrm{T}} v_{3}=0 ; \\
& h_{4}=\omega_{4}{ }^{\mathrm{T}} v_{4}=0 ; h_{5}=\omega_{5}{ }^{\mathrm{T}} v_{5}=0 ; h_{6}=\omega_{6}{ }^{\mathrm{T}} v_{6}=0 ;
\end{aligned}
$$

All the axes for the other twists can be further obtained as

$$
\begin{aligned}
& l_{2}=\left\{\left[\lambda, 0, C_{Y, F_{Y}} / C_{\theta_{X}, F_{Y}}\right]^{\mathrm{T}}: \lambda \in \mathbf{R}\right\} \\
& l_{3}=l_{6}=\left\{[0,0, \lambda]^{\mathrm{T}}: \lambda \in \mathbf{R}\right\} \\
& l_{4}=\left\{\begin{array}{l}
\left.\left[\lambda, 0, C_{Y, M_{X}} / C_{\theta_{X}, M_{X}}\right]^{\mathrm{T}}: \lambda \in \mathbf{R}\right\} \\
l_{5}=\left\{\left[0, \lambda,-C_{X, M_{Y}} / C_{\theta_{Y}, M_{Y}}\right]^{\mathrm{T}}: \lambda \in \mathbf{R}\right\}
\end{array}\right.
\end{aligned}
$$

In order to validate that the actual rotation center is very close to Point $O_{R C}$, let $l=12.5 \mathrm{~mm}$, $D=1.5 \mathrm{~mm}, \theta=45^{\circ}$, and $\left|O O_{1}\right|=10 \mathrm{~mm}$. The material for the mechanism is chosen as nylon PA 2200 with a Young's Modulus of $1646 \mathrm{MPa}$ and a Poisson's ratio of 0.33. From Equations (1)-(11), we obtain the factors of the compliance matrix:

$$
\begin{aligned}
& C_{X, F_{X}}=C_{Y, F_{Y}}=3.1929 \times 10^{-5}(\mathrm{~m} / \mathrm{N}) \\
& C_{X, M_{Y}}=C_{Y, M_{X}}=2.956 \times 10^{-3}(\mathrm{~m} / \mathrm{Nm}) \\
& C_{\theta_{X}, F_{Y}}=C_{\theta_{Y}, F_{X}}=-2.956 \times 10^{-3}(\mathrm{rad} / \mathrm{N}) \\
& C_{\theta_{X}, M_{X}}=C_{\theta_{Y}, M_{Y}}=3.1459 \times 10^{-1}(\mathrm{rad} / \mathrm{Nm})
\end{aligned}
$$

Because all the pitches of the six twists are zero, the motions of the end-effector can be regarded as pure rotations about the axes $l_{1}-l_{6}$ of the six twists. The real twist axes $l_{1}-l_{6}$ and the ideal twist axes are illustrated in Figure 6. The distance between the real twist axes and ideal twist axes is

$$
\mid \begin{aligned}
& 2.956 \times 10^{-3} / 3.1459 \times 10^{-1}-10 \times 10^{-3} \mid=0.6 \times 10^{-3} \mathrm{~m} \\
& \left|3.1929 \times 10^{-5} / 2.956 \times 10^{-3}-10 \times 10^{-3}\right|=0.8 \times 10^{-3} \mathrm{~m}
\end{aligned}
$$

which is defined as the center shift or the parasitic error of the remote center of the RCC mechanism $[15,17]$. It can be seen from Figure 6 that the real twist axis does not overlap with the ideal twist axis, which goes through point $O_{R C}$, and the distance between the two axes is $0.6 \mathrm{~mm}$ (or 0.8 $\mathrm{mm}$ ), which cannot be neglected in precision-required applications. 


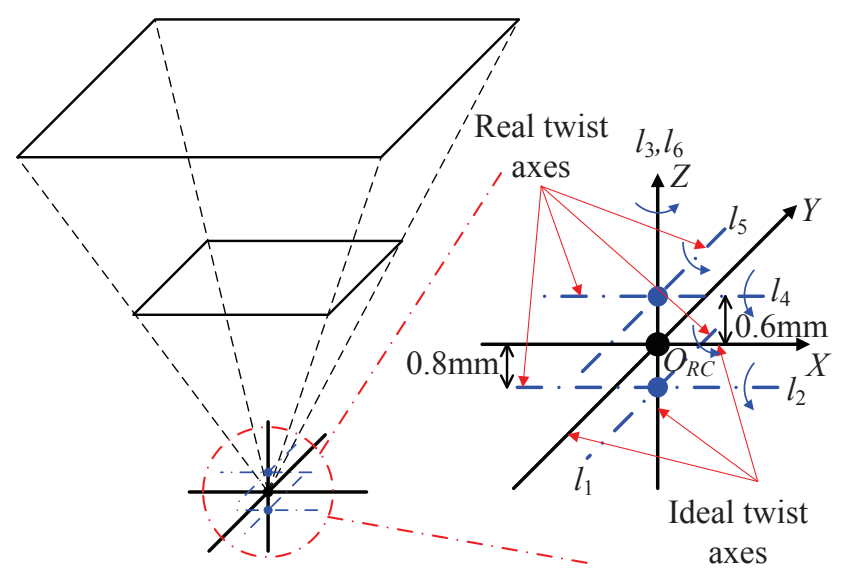

Figure 6. Illustration for real twist axes and ideal twist axes.

\section{Finite Element Analysis Validation}

Finite element analysis (FEA) was performed to validate the analytical model of the RCC mechanism. The ANSYS Workbench finite element software was utilized to construct the FEA model of the mechanism. In order to validate the efficiency of the analytical model sufficiently, three samples with different geometrical parameters were set for the comparisons with FEA results, as shown in Table 1. The material of the mechanism was selected as nylon PA 2200 with a Young's Modulus of $1646 \mathrm{MPa}$ and a Poisson's ratio of 0.33 . The three-dimensional (3D) model was generated by Solidworks as a monolithic part, and was subsequently imported to ANSYS in parasolid format. The 3D tetrahedron element was used to mesh this model, and the upper rigid body of the mechanism was fixed.

Table 1. Geometrical parameters of different samples.

\begin{tabular}{ccccc}
\hline Sample & $\boldsymbol{l}(\mathbf{m m})$ & $\boldsymbol{D}(\mathbf{m m})$ & $\boldsymbol{\theta}\left({ }^{\circ}\right)$ & $\left|\boldsymbol{O O}_{\mathbf{1}}\right|(\mathbf{m m})$ \\
\hline 1 & 12.5 & 1.5 & 45 & 10 \\
2 & 15 & 2 & 60 & 15 \\
3 & 20 & 1.2 & 30 & 20 \\
\hline
\end{tabular}

The compliance factors in the compliance matrix shown in Equation (11) were obtained using the static analysis module of the ANSYS Workbench. By respectively applying the four different sets of forces (lateral forces $F_{X}, F_{Y}$, pure moments $M_{X}, M_{Y}$, vertical forces $F_{Z}$, and pure moments $M_{Z}$ ) at Point $O$, the corresponding displacements of Point $O$ from the FEA results (shown in Figure 7) can be used to calculate the compliance factors. All of the applied loads and moments were $1 \mathrm{~N}$ and $1 \mathrm{Nm}$, respectively, in FEA. The compliance factors calculated from the analytical model and the FEA model are listed in Table 2. The analytical results and FEA results are in good agreement. The relative errors between the two results are less than $8 \%$. The maximum error of $7.83 \%$ between the two results is mainly caused by the strong non-linearities of beam flexure when the end-effector undergoes the $Z$ axis force. 


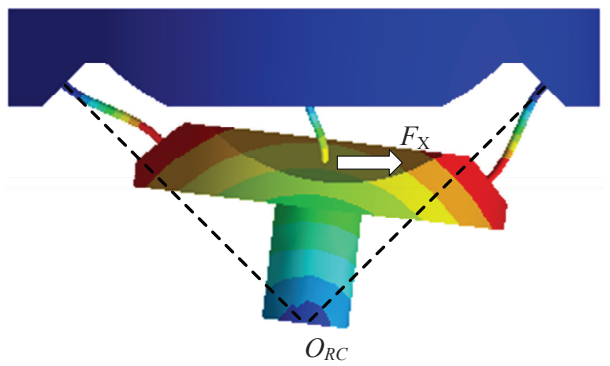

(a)

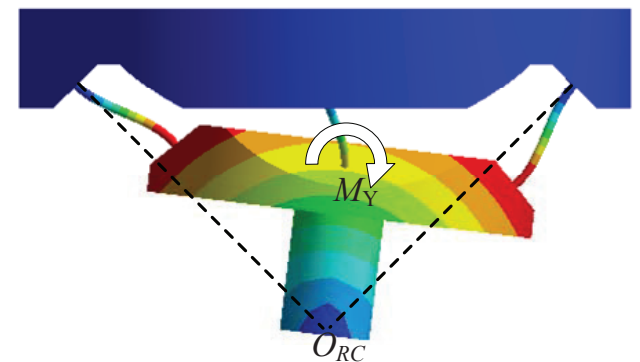

(b)

Figure 7. Finite element analysis (FEA) results of the RCC mechanism in different loading cases of (a) Direction fixed force $F_{X}$ and (b) Pure Moment $M_{Y}$.

Table 2. Comparison of the compliance factors between analytical (An) and Finite element analysis (FEA) results.

\begin{tabular}{|c|c|c|c|c|c|c|c|c|c|}
\hline \multirow[b]{2}{*}{ Compliance factors } & \multicolumn{3}{|c|}{ Sample 1} & \multicolumn{3}{|c|}{ Sample 2} & \multicolumn{3}{|c|}{ Sample 3} \\
\hline & An & FEA & Err (\%) & An & FEA & Err $(\%)$ & An & FEA & $\operatorname{Err}(\%)$ \\
\hline$C_{X, F_{X}}, C_{Y, F_{Y}}(\mu \mathrm{m} / \mathrm{N})$ & 31.93 & 32.23 & 0.93 & 29.93 & 31.43 & 4.77 & 188.9 & 183.5 & 2.94 \\
\hline$C_{X}, M_{Y}, C_{Y, M_{X}}(\mathrm{~mm} / \mathrm{Nm})$ & 2.96 & 3.07 & 3.58 & 1.08 & 1.13 & 4.42 & 15.9 & 15.4 & 3.25 \\
\hline$C_{Z, F_{Z}}(\mu \mathrm{m} / \mathrm{N})$ & 2.12 & 2.30 & 7.83 & 0.96 & 1.04 & 7.69 & 10.63 & 11.11 & 4.32 \\
\hline$C_{\theta_{X}}, F_{Y}, C_{\theta_{Y}, F_{X}}(\mathrm{mrad} / \mathrm{N})$ & 2.96 & 3.08 & 3.89 & 1.08 & 1.13 & 4.42 & 15.9 & 15.4 & 3.25 \\
\hline$C_{\theta_{X}}, M_{X}, C_{\theta_{Y}, M_{Y}}(\mathrm{mrad} / \mathrm{Nm})$ & 314.6 & 330.3 & 4.75 & 47.35 & 50.56 & 6.35 & 1391.8 & 1352.3 & 2.92 \\
\hline$C_{\theta_{Z}, M_{Z}}(\mathrm{mrad} / \mathrm{Nm})$ & 452.2 & 469.3 & 3.64 & 147.8 & 153.6 & 3.78 & 1163.6 & 1136.7 & 2.37 \\
\hline
\end{tabular}

\section{Experimental Verification}

To validate the analytical model, experimental tests were also conducted on a prototype of the RCC mechanism with $l=12.5 \mathrm{~mm}, D=1.5 \mathrm{~mm}, \theta=45^{\circ}$, and $\left|O O_{1}\right|=10 \mathrm{~mm}$, which can be rapidly manufactured by 3D laser printing technique (selective laser sintering, SLS). The chosen material of the prototype was nylon PA 2200 with a Young's modulus of $1646 \mathrm{MPa}$ and the Poisson's ratio of 0.33 . The photography of the prototype is shown in Figure 8a. A digital dial gage ( $1 \mu \mathrm{m}$ resolution) was employed to measure the displacement, while a series of standard weights were used to apply the pull forces to the end-effector of the RCC mechanism.

The experimentation includes two steps. In the first step (Figure $8 \mathrm{~b}$ ), the applied force generated by the weights was parallel to the $Z$ axis of the mechanism. As the applied force does not pass through the $Z$ axis, a $Z$ directional force and a $Y$ directional moment can be generated. The dial gage can measure displacement at a certain point, and the displacement originated from the $Z$ axial compression was neglected, so $C_{\theta_{X}}, M_{X}$ and $C_{\theta_{Y}}, M_{Y}$ can be obtained from the test.

In the second step (Figure 8c), the applied force generated by the weights was vertical to the $Z$ axis of the mechanism. The digital dial gage is firstly pre-compressed, and then released after the force is applied. As the force does not pass through the end point $O$, the variations of $X$ directional force and $Y$ directional moment can be caused by the hanging weights. Due to the small deformation of the RCC mechanism, the movements of the fixtures used to hang the weights can be neglected. Therefore, the theoretical applied force and moment can be calculated by the known hanging weights and moment arm. Both the applied force and the corresponding displacement at a certain point can be known from the measurement condition. $C_{\theta_{X}}, M_{X}$ is obtained from the first test, and $C_{X}, M_{Y}$ is equal to $C_{\theta_{Y}}, F_{X}$, so, $C_{X}, F_{X}$ and $C_{X, M_{Y}}$ can be calculated in two measurements at different applying and measurement points [25]. 


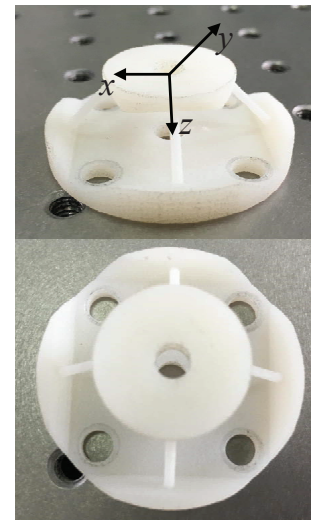

(a)

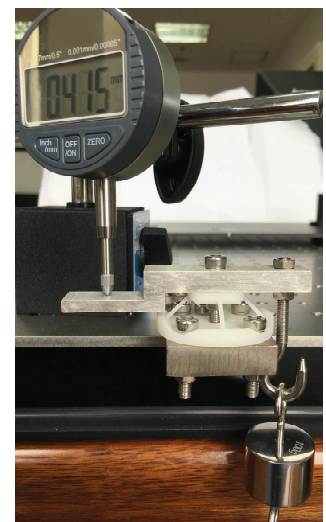

(b)

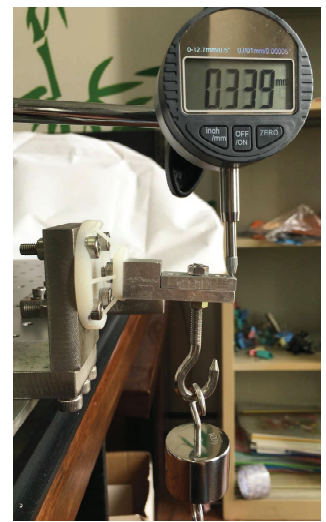

(c)

Figure 8. The experimental setups. (a) Photo of the prototype, and experimental conditions of (b) the first step and (c) the second step.

Each step of the experimentation process was repeated three times. The experimental results obtained from the three repeats was averaged to the final values of the compliances. The comparisons of analytical and experimental results are listed in Table 3. It can be seen that the errors between the three results are all small enough to verify the effectiveness of the proposed analytical model.

Table 3. Comparisons of compliance factors between analytical and experimental results.

\begin{tabular}{cccc}
\hline Compliance Factors & Analytical & Experimental & Error (\%) \\
\hline$C_{X}, F_{X}, C_{Y, F_{Y}}(\mu \mathrm{m} / \mathrm{N})$ & 31.93 & 32.78 & 2.59 \\
$C_{X}, M_{Y}, C_{Y, M_{X}}(\mathrm{~mm} / \mathrm{Nm})$ & 2.96 & 3.18 & 6.91 \\
$C_{\theta_{X}}, F_{Y}, C_{\theta_{Y}, F_{X}}(\mathrm{mrad} / \mathrm{N})$ & 2.96 & 3.18 & 6.91 \\
$C_{\theta_{X}, M_{X}}, C_{\theta_{Y}}, M_{Y}(\mathrm{mrad} / \mathrm{Nm})$ & 314.6 & 311.8 & 0.9 \\
\hline
\end{tabular}

\section{Performance Analysis}

The analytical model was established to analyze the influences of the geometric parameters and the material properties on the compliance factors and rotation center shifts. The designed geometric parameters of the mechanism were chosen as $l, D, \theta$, and $\left|O O_{1}\right|$. It can be noticed that all compliance factors of the mechanism vary inversely with the elastic Young's modulus $E$, and increase with the increase of $l$ and the decrease of $D$.

To evaluate the influences of the other two parameters $\theta$ and $\left|O O_{1}\right|$ on the compliance factors, three dimensional plots of all compliance factors in terms of the two geometric parameters mentioned above are shown in Figure 9. Each time that the two parameters are varied, the other parameters are considered to be constant. These constant values are: $E=1646 \mathrm{MPa}, l=12.5 \mathrm{~mm}, D=1.5 \mathrm{~mm}$. It can be seen from Figure 9 that the two geometric parameters also have significant effects on the compliance factors, and several conclusions can be derived as follows to provide a useful and accurate reference for the design and manufacture of satisfactory RCC mechanism structures:

(1) The compliance factors $C_{X, F_{X}}$ increase when $\theta$ increases. The variation tendency of $C_{X,}, F_{X}$ versus $\left|O O_{1}\right|$ is dependent on $\theta$.

(2) $\left|O O_{1}\right|$ has no influence on the vertical compliance factor $C_{Z, F_{Z}}$, which increases sensitively with the decrease of $\theta . C_{\theta_{X}}, M_{X}$ decreases when the two parameters increase.

(3) $C_{X, M_{Y}}$ varies slightly with $\left|O O_{1}\right|$, and presents a maximum in terms of $\theta$, around $\theta=45^{\circ}$. 


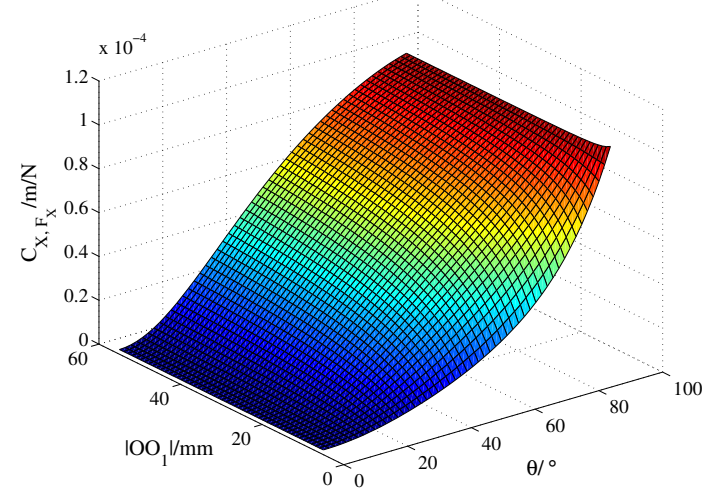

(a)

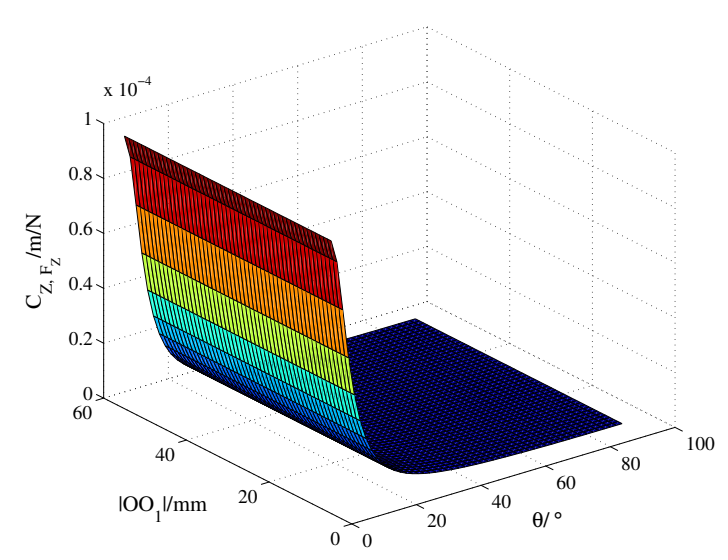

(c)

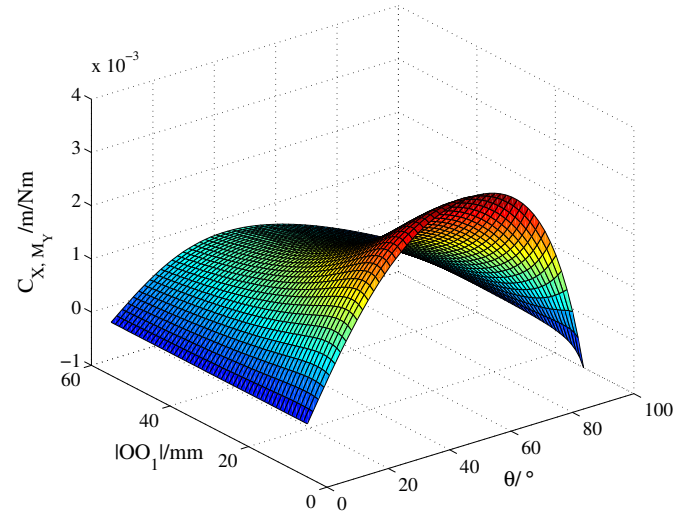

(b)

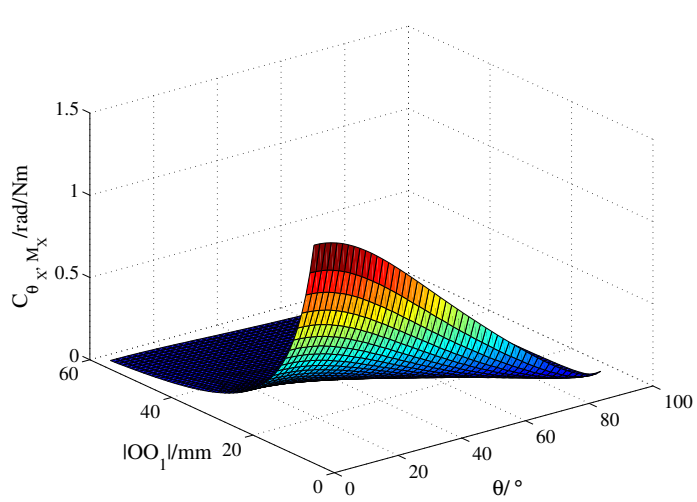

(d)

Figure 9. Compliance factors plots in terms of mechanism geometry. (a) Compliance $C_{X, F_{X}}$; (b) Compliance $C_{X, M_{X}} ;(\mathbf{c})$ Compliance $C_{Z, F_{Z}}$ (d) Compliance $C_{\theta_{X}}, M_{X}$.

The influences of different geometric parameters of the mechanism on the rotation center shift were also analyzed by the proposed analytical model. Although the real rotation center is very close to the ideal rotation center based on the aforementioned analytical results, the RCC mechanism is expected to minimize its center shift if it is designed for precise rotation. According to Equation (25), the locations of rotation center are the ratio functions of the compliance factors; hence, the influences of Young's modulus $E$ of the material on the center shift are removed. The variation tendencies of the center shift with respect to the changing dimensions are all graphically represented in Figure 10. As can be seen from Figure 10a-d, the dimensions of the beam flexure have a significant influence on the center shift. The center shifts in both loading cases (direction fixed force $F_{X}, F_{Y}$ and pure Moment $M_{X}, M_{Y}$ ) decrease with the increase of $l$ and the decrease of $D$, and vary quasi-linearly with $\left|O O_{1}\right|$. It can be concluded from Figure 10 that the minimization of the center shifts may be achieved by increasing $l$ or reducing $D,\left|O O_{1}\right|$, and $\theta$. 


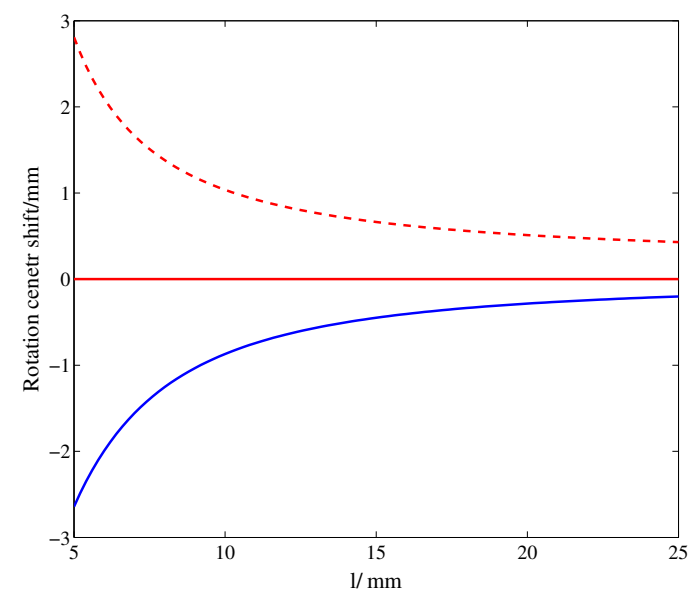

(a)

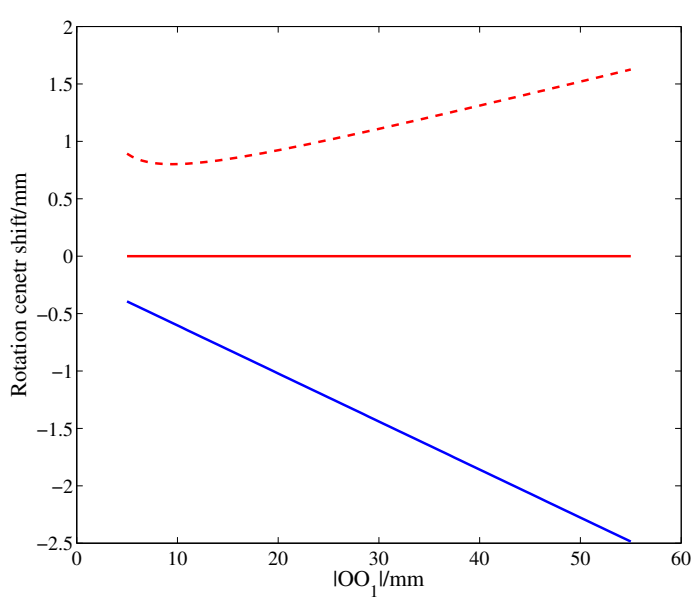

(c)

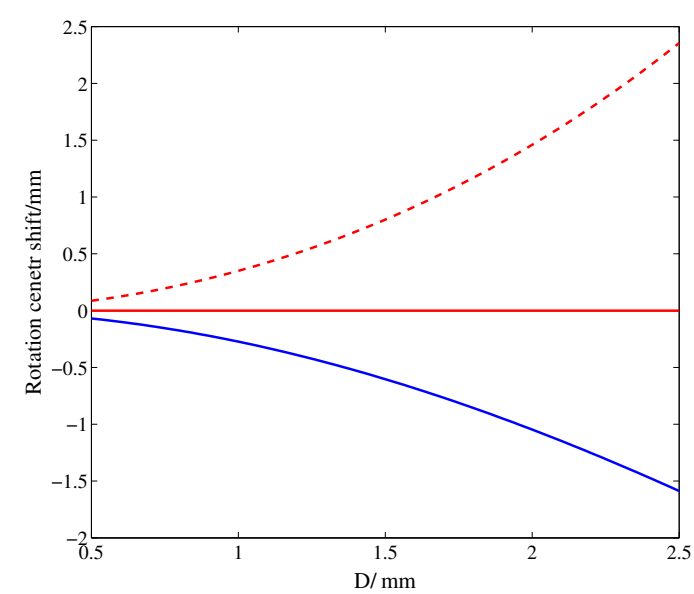

(b)

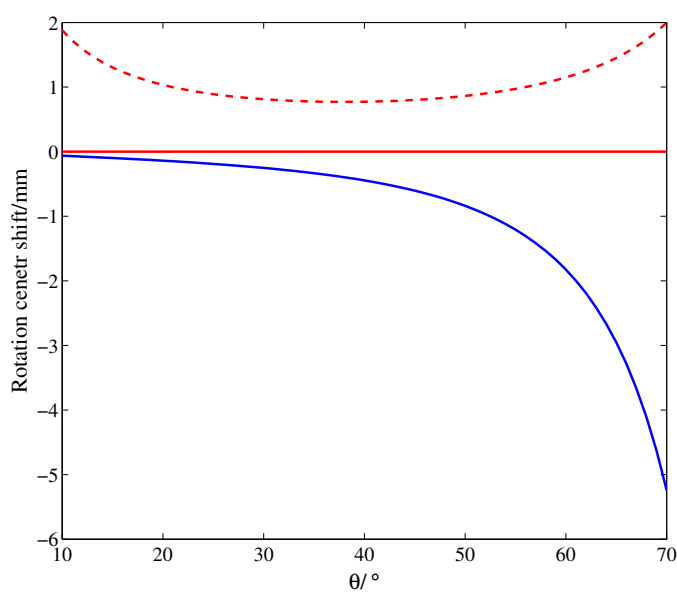

(d)

Figure 10. The influences of different RCC mechanism parameters on the center shift (solid-pure moment loading case, dot-direction fixed force loading case. (a) influence of $l$; (b) influence of $D$; (c) influence of $\left|O O_{1}\right| ;(\mathbf{d})$ influence of $\theta$.

\section{Conclusions}

In this paper, an analytical model for a class of RCC mechanisms has been made in order to evaluate the compliance properties and rotational precision of the mechanism. The RCC mechanism analyzed in this paper combines two sets of isosceles-trapezoidal flexure pivots in a parallel manner to realize 3-DOFs rotation around a fixed remote center. The stiffness matrix approach was used to model the statics of the compliant mechanism, providing the close-form equations of the compliance factors for the compliant mechanism. The rotation center locations of the RCC mechanism were also analyzed using the analytical model and screw theory to evaluate the rotational precision of the mechanism. In order to verify the analytical model, both FEA simulations and experimental tests were performed. The comparison shows that the FEA, experimental, and analytical results agree with each other with a deviation of less than $8 \%$. Furthermore, the influences of geometric parameters on the compliance factors and the rotation center locations were represented graphically, providing a theoretical basis for practical design. 
Acknowledgments: This work was supported by the National Natural Science Foundation of China (Grant No. 51605275) and Shanghai Municipal Education Commission (Grant No. ZZGCD15085 and No. ZZGCD15086).

Author Contributions: Lei-Jie Lai established the analytical model, conceived and designed the experiments and wrote the paper; Zi-Na Zhu drew the 3D structure and performed the finite element analysis.

Conflicts of Interest: The authors declare no conflict of interest.

\section{References}

1. Choi, K.B.; Lee, J.J. Passive compliant wafer stage for single-step nano-imprint lithography. Rev. Sci. Instrum. 2005, 76, 075106.1-075106.6.

2. Choi, B.J.; Sreenivasan, S.V.; Johnson, S.; Colburn, M.; Wilson, C.G. Design of orientation stages for step and flash imprint lithography. Precis. Eng. 2001, 25, 192-199.

3. Lan, H.B.; Ding, Y.C.; Liu, H.Z.; Lu, B.H. Development of a step micro-imprint lithography tool. J. Micromech. Microeng. 2007, 17, 2039-2048.

4. Simeone, F.C.; Albonetti, C.; Cavallini, M. Progress in Micro- and Nanopatterning via Electrochemical Lithography. J. Phys. Chem. C 2009, 113, 18987-18994.

5. Ahmed, N. Towards Active Monitoring of the Micro Transfer Printing Process. Master's Thesis, University of Illinois at Urbana-Champaign, Urbana, IL, USA, 2011.

6. Drake, S.H.; Simunovic, S.N. Compliant Assembly System Device. US Patent 4155169, 22 May 1979.

7. Watson, P.C. Remote Center Compliance System. US Patent 4098001, 4 July 1978.

8. Nakao, M.; Sugiyama, S.; Hatamura, Y.; Hamaguchi, T. Remote-centered compliance mechanism of micro-suspension for contact recording head. IEEE-ASME Trans. Mechatron. 1996, 1, 245-249.

9. Zhao, F.; Wu, P.S.Y. VRCC: A variable remote center compliance device. Mechatronics 1998, 8, 657-672.

10. Lee, S.; Won, S.; Choi, S. Development of a new variable remote center compliance for assembly robots. Adv. Robot. 2000, 14, 241-255.

11. Hiroshima, H.; Komuro, M.; Kurashima, Y.; Kim, S.H.; Muneishi, T. Step-and-Repeat Photo-Nanoimprint System Using Active Orientation Head. Jpn. J. Appl. Phys. 2004, 43, 4012-4016.

12. Qu, J.; Chen, W.; Zhang, J. A parallelogram-based compliant remote-center-of-motion stage for active parallel alignment. Rev. Sci. Instrum. 2014, 85, 095112.

13. Qu, J.; Chen, W.; Zhang, J.; Chen, W. A piezo-driven 2-DOF compliant micropositioning stage with remote center of motion. Sens. Actuators A: Phys. 2016, 239, 114-126.

14. Ciblak, N.; Lipkin, H. Design and Analysis of Remote Center of Compliance Structures. J. Rob. Syst. 2003, 20, 415-427.

15. Pei, X.; Yu, J.J.; Zong, G.H.; Bi, S.S.; Yu, Z.W. Analysis of Rotational Precision for an Isosceles-Trapezoidal Flexural Pivot. ASME J. Mech. Des. 2008, 130, 052302.

16. Pei, X.; Yu, J.J.; Zong, G.H.; Bi, S.S. The stiffness model of leaf-type isosceles-trapezoidal flexural pivots. ASME J. Mech. Des. 2008, 130,1-6.

17. Su, H.J.; Shi, H.; Yu, J. A Symbolic Formulation for Analytical Compliance Analysis and Synthesis of Flexure Mechanisms. ASME J. Mech. Des. 2012, 134,1-9.

18. Hopkins, J.B.; Culpepper, M.L. Synthesis of multi-degree of freedom, parallel flexure system concepts via Freedom and Constraint Topology (FACT) Part I: Principles. Precis. Eng. 2010, 34, 259-270.

19. Yu, J.; Li, S.; Su, H.J.; Culpepper, M.L. Screw Theory Based Methodology for the Deterministic Type Synthesis of Flexure Mechanisms. Precis. Eng. 2011, 3, 1-14.

20. Choi, K.B.; Kim, D.H. Monolithic parallel linear compliant mechanism for two axes ultraprecision linear motion. Rev. Sci. Instrum. 2006, 77, 1-7.

21. Hao, G.; Kong, X. A normalization-based approach to the mobility analysis of spatial compliant multi-beam modules. Mech. Mach. Theory 2013, 59, 1-19.

22. Lobontiu, N.; Garcia, E.; Hardau, M.; Bal, N. Stiffness Characterization of Corner-Filleted Flexure Hinges. Rev. Sci. Instrum. 2004, 75, 4896-4905.

23. Dai, J.S.; Ding, X. Compliance analysis of a three-legged rigidly-connected platform device. ASME J. Mech. Des. 2006, 128, 755-764. 
24. Murray, R.M.; Li, Z.; Sastr, S.S. A Mathematical Introduction to Robotic Manipulation; CRC Press: Boca Raton, FL, USA, 1994.

25. Lin, R.; Zhang, X.; Long, X.; Fatikow, S. Hybrid flexure hinges. Rev. Sci. Instrum. 2013, 84, 085004.

(C) 2016 by the authors; licensee MDPI, Basel, Switzerland. This article is an open access article distributed under the terms and conditions of the Creative Commons Attribution (CC-BY) license (http://creativecommons.org/licenses/by/4.0/). 